\title{
Prevalence and Risk Factors Leading to Acute Respiratory Failure among Critically III Patients at Assiut University Hospital
}

\author{
Azza Farhan Amer ${ }^{1}$, Amany Omar Mohamed ${ }^{2}$ \& Mervat Anwar Abd El-Aziz ${ }^{3}$. \\ 1. A Specialist Nursing In Al-Azher University, Egypt. \\ 2. Professor, Department of Chest Diseases, Faculty of Medicine, Assiut University, Egypt. \\ 3. Assistant Professor of Critical care \& emergency Nursing Faculty of Nursing, Assuit University, Egypt.
}

\begin{abstract}
Background: Acute respiratory failure in chronic respiratory disease remains a major reason for mechanical ventilation in ICU patients. Aim of study: to identify prevalence and risk factors of acute respiratory failure among critically ill patients at Assiut University Hospital. Subject and Method: All patients diagnosed with acute Respiratory failure within 6 months period admitted in to chest intensive care unit and trauma at Assiut University Hospital. Tools of data collection: Tool (1) Acute respiratory failure patient assessment sheet, Tool (2) outcomes of respiratory failure. Results: Risk factors of acute respiratory failure among critically ill patients in the above mentioned setting at Assuit University Hospital was 150 patients who exposed to respiratory failure. About that $90.8 \%$ in the chest ICU groups were smoking and $76.7 \%$ were suffer from COPD but Injury of spine, brain or chest and smoking were the majority risk factors in the trauma ICU groups (50\% and 43,3\% respectively). With statistical significant differences between both groups $\left(\mathrm{p}=0.000^{*}\right)$.Conclusion: There was highly statistical significant differences relationship between chest ICU groups and trauma ICU groups in smoking, COPD, occupation and the prevalence of Acute respiratory failure with $(\mathrm{p}=0.000 *)$. Recommendations: Application of life style modification for modifying risk factors leading to ARF.
\end{abstract}

\section{Key words: Acute Respiratory Failure, Prevalence \& Risk Factors.}

\section{Introduction}

Respiratory failure is one of the most communal reasons for admission to the intensive care unit (ICU) and a co morbidity in patients acknowledged for acute care. What's more, it's the leading cause of death from pneumonia and chronic obstructive pulmonary disease (COPD) in the United States. (Pfister et al., 2016).

In this respect, Leclair and Allen, 2018 reported that, respiratory failure is a clinical circumstance that happens when the respiratory system fails to maintain its main function which is gas exchange, in which $\left(\mathrm{paO}_{2}\right)$ partial pressure of oxygen lower than $60 \mathrm{mmHg}$ and $\left(\mathrm{PaCO}_{2}\right)$ partial pressure of carbon dioxide higher than $50 \mathrm{mmHg}$.may be acute or chronic. In acute failure, life-threatening confusions in arterial blood gases (ABGs) and acid-base status occur, and patients may need immediate intubation.

Clinical indicators of acute respiratory disappointment include, partial pressure of arterial oxygen $\left(\mathrm{PaO}_{2}\right)$ below $60 \mathrm{~mm} \mathrm{Hg}$, or arterial oxygen saturation as measured by pulse oximetry $\left(\mathrm{Spo}_{2}\right)$ below $91 \%$ on room air, $\mathrm{Paco}_{2}$ above $50 \mathrm{~mm} \mathrm{Hg}$ and pH below 7.35 (Malay, et al., 2017).

Respiratory failure is categorized according to blood gases abnormalities in to type I and type II. Hypoxemic respiratory failure (type 1) in which $\mathrm{paO}_{2}<60 \mathrm{mmHg}$ with normal $\mathrm{paco}_{2}$. In this type the gas exchange is decreased at the level of alveolocapillary membrane. Hypercapnia respiratory failure (type 2) in which $\mathrm{paco}_{2}>50 \mathrm{mmHg}$,is result of inadequate alveolar ventilation secondary to decreased ventilator ambition, respiratory muscle fatigue or failure and increased work of breathing (Craig et al., 2016).

Incidence and prevalence of acute respiratory failure.

Incidence: about 360,000 cases per year in the unit states, 36\% die during hospitalization, morbidity and mortality rates increase with age and presence of co morbidities (Rahul et al., 2017).

Respiratory failure may be due to pulmonary or extra-pulmonary causes which include central nervous system causes due to depression of the neural drive to breath as in cases of overdose of narcotic and sedative, disorders of peripheral nervous system: respiratory muscle and chest wall weakness as in cases of Guillian-Barre syndrome and myasthenia gravis. Upper and lower airways obstruction: due to various causes as in suitcases of exacerbation of chronic pulmonary diseases and sever bronchial asthma.(Mc Donell et al., (2015).

Injury to the spinal card or brain can proximately affect your breathing. An injury to the ribs or chest can also hamper the breathing process. Acute respiratory distress syndrome (ARDS), Drug or alcohol misuse if you over dose on drug or drink too much alcohol you can impair brain function, chemical inhalation in inhaling toxic chemical, 
smoke or fumes also cause acute respiratory failure (Phuong \& Virginia, 2014).

High-risk acute respiratory failure increase with smoke tobacco harvests, drink alcohol excessively, have family history of respiratory disease or condition, sustain an injury to the spine, brain or chest, have chronic (long term) respiratory problem such as cancer of the lungs, COPD or asthma, cardiovascular complaints; heart failure, pulmonary embolism, allergic disorders, bronchospasm, increased oxygen request fever, infection, mechanical issue pneumothorax, pleural effusion, abdominal distention, inspiratory muscle fatigue (Patricia \& Dorrie, 2018)

Nursing care and concerted administration the over goal for patient in acute respiratory failure include ABG values within the patient's base line, active cough and ability to clear secretion. (Michelle, 2014).

\section{Significance of the study}

Acute respiratory failure(ARF) endure a major cause of morbidity and mortality in the intensive care location, ARF may be responsible for as many as $10 \%$ to $15 \%$ for admissions to medical ICU and for as many as $50 \%$ to $75 \%$ of those patients who require ICU stays longer than 7 days. (Pradeep, et al., 2016) Acute respiratory failure grounds numerous complications such as pulmonary embolism, reduced cardiac output, arrhythmias, acute kidney injury, incidence of acute respiratory failure admitted to chest department is ranged 500 patients in the year 2016 affording to the statistical record of Assiut University Hospital

\section{Aim of the study \\ The present study aims to}

Identify the prevalence and risk factors of acute respiratory failure among critically ill patients.

\section{Research question:}

What is the prevalence and risk factors of acute respiratory failure among critical ill patients at Assiut university hospital?

\section{Patients \& Method}

\section{Research design}

Descriptive research design was used to conduct this study.

\section{Setting}

This study was carried out in chest intensive care unit and trauma intensive care unit at Assiut university hospital.

\section{Sample}

All patients diagnosed with Acute Respiratory failure who defined based on need for Endotracheal intubation and mechanical ventilator support for at least 24 hours, This sample was assigned to two groups of patients with acute respiratory failure group one $n=120$ patient admitted to chest ICU, group two $\mathrm{n}=30$ patient admitted to trauma ICU diagnosed with Acute Respiratory failure were included in the study for consecutive six month. The sample was unsamiliar because in the specific period for data collection, the number of patients admitted into chest ICU $n=120$ and the patients admitted into trauma ICU $\mathrm{n}=30$.

Inclusion criteria

The study included patients had the following criteria:

1- Two types of Acute Respiratory failure Hypoxemic respiratory failure, Hypercapnice respiratory failure.

2- Aged between 20-60 years old, both sexes will be included in this study.

\section{Exclusion criteria}

The study excluded patients had the following criteria:

1- Requirement for emergent cardiopulmonary resuscitation.

2- Home mechanical ventilation or oxygen longterm supplementation.

3- Severe disease with a low expectation of life.

\section{Tools of data collection}

Two tools used by researcher in this study after reviewing of the related literature.

Tool one: Acute respiratory failure patient's assessment Sheet:

This tool was developed by the researcher after reviewing the related literature. Aimed to assess patient with acute respiratory failure among critical ill patient. Contained two parts:

\section{Part I. Patient profile}

This part included patient's characteristics and clinical data such as patient name, age, sex, patient's diagnosis, past medical history and surgical problem. date of ICU admission, date of ICU discharge, need for mechanical ventilation, period of his hospital stay, died and survival of the patient.

In additional to, assessment arterial blood gases \& oxygen saturation.

\section{Part II: Hemodynamic monitoring}

This part included respiratory rate, heart rate, pulse rate, temperature, Blood pressure monitoring, Mean arterial blood pressure, and oxygen saturation. In additional to to mechanical ventilator parameters.

Tool two: A cute respiratory failure's patient outcome:

This tool consists of four main categories as follows:

$>$ ICU stay.

$>$ Mortality rate

$>$ Duration of mechanical ventilation.

$>$-Occurrence of complications 


\section{Method}

The study was conducted throughout three main phases, preparatory phase, implementation phase and evaluation phase.

\section{1-Perparatory phase}

- A written permission was obtained from authorized administration, faculty of nursing, head of chest department at Assiut university hospital and head of anesthesia department at Assiut university hospital to collect data from previously mentioned research setting after explanation the purpose and nature of the study.

- Confidentiality of data, privacy, voluntary participation and right to refuse to participate in the study were emphasized to patient's responsible person.

- The tools (I and II ) used in this study were developed by researcher after reviewing of current and international related literature in a various Aspects of this study using books and magazines was done.

- Content validity of the developed tools ( I and II ) were tested the content validity by a jury of (7) specialists in the field of critical care nursing and intensive care medical, the necessary modification were done.

- A pilot study was carried out to assess tool clarity, understandability, and applicability of the study tools. Moreover, to identify problems that may be encountered during the actual data collection. It applied on $10 \%$ patients from the entirely sample in a selected setting to check clarity and understanding of the study tool after the necessary modification were done. The pilot study patients were included in the study sample.

- The Reliability was done on the developed tools using Cronach's Alpha and reliability level was 0.87 to assess the consistency and stability of the tools.

\section{Ethical considerations}

1- Research proposal was approved from Ethical committee in the Faculty of nursing.

2- There is no risk for study subject during application of the research.

3- The study was following common ethical principles in clinical research.

4- Written consent was obtained from patients or guidance that is willing to participate in the study, after explaining the nature and purpose the study.

5- Confidentiality and anonymity was assured.

6- Study subject have the right to refuse to participate and or withdraw from the study without any rational any time.

7- Study subject privacy was considered during collection of data.

\section{2-Implementation phase}

Once permission was granted to proceed with proposed study the researcher initiated data collection.

\section{Data collection}

- Data were collected in six months approximately.

- Started from the first of January 2018 until the end of June 2018.

- The data were collected from the first day of admission after stabilization of the patient's condition and at 2 nd day of patient admission and then data recorded in the developed tools.

- The researcher assigned study sample (150 patients) to two groups (group one 120 patients, group two 30 patients).

- Incidence of Acute respiratory failure will be identified from total admitted patients in ICUs.

- Every patients diagnosed with acute respiratory failure will be included in the study.

- All patients (diagnosed with Acute Respiratory failure) admitted in the previous mentioned ICUs were assessed regarding Patient characteristics and clinical data by using tool one part I.

- All patients (diagnosed with Acute Respiratory failure) were assed their heart rate, respiratory rate and blood pressure by using tool one part II .

Statistical analysis: Date entry and data analysis were done using SPSS version 19 (Statistical Package for Social Science). Data were presented as number, percentage, mean, standard deviation. Chi-square test and Fisher Exact test were used to compare between qualitative variables. Independent samples t-test was used to compare quantitative variables between two groups. Mann-Whitney test was used to compare quantitative variables between groups in case of nonparametric data. P-value considered statistically significant when $\mathrm{P}<0.05$. 


\section{Results}

Table (1): Percentage distribution of studied patients regarding socio-demographic characteristics $(n=150)$.

\begin{tabular}{|c|c|c|c|c|c|}
\hline \multirow[t]{2}{*}{ Variables } & \multicolumn{2}{|c|}{$\begin{array}{c}\text { Chest ICU } \\
(n=120)\end{array}$} & \multicolumn{2}{|c|}{$\begin{array}{c}\text { Trauma ICU } \\
(n=30)\end{array}$} & \multirow[t]{2}{*}{ P-value } \\
\hline & No. & $\%$ & No. & $\%$ & \\
\hline \multicolumn{5}{|l|}{ Age: (years) } & \multirow{4}{*}{$0.003 *$} \\
\hline$<40$ & 38 & 31.7 & 17 & 56.7 & \\
\hline $40-<50$ & 45 & 37.5 & 2 & 6.7 & \\
\hline$\geq 50$ & 37 & 30.8 & 11 & 36.7 & \\
\hline Mean \pm SD & \multicolumn{2}{|c|}{$51.16 \pm 9.17$} & \multicolumn{2}{|c|}{$42.87 \pm 11.74$} & $0.005^{*}$ \\
\hline \multicolumn{5}{|l|}{ Sex: } & \multirow{3}{*}{0.353} \\
\hline Male & 73 & 60.8 & 21 & 70.0 & \\
\hline Female & 47 & 39.2 & 9 & 30.0 & \\
\hline \multicolumn{5}{|l|}{ Occupation: } & \multirow{5}{*}{$0.000 *$} \\
\hline Employee & 34 & 28.3 & 9 & 30.0 & \\
\hline Farmer & 41 & 34.2 & 3 & 10.0 & \\
\hline Housewife & 43 & 35.8 & 8 & 26.7 & \\
\hline Unemployed & 2 & 1.7 & 10 & 33.3 & \\
\hline \multicolumn{5}{|l|}{ Marital status: } & \multirow{3}{*}{0.261} \\
\hline Married & 117 & 97.5 & 28 & 93.3 & \\
\hline Single & 3 & 2.5 & 2 & 6.7 & \\
\hline
\end{tabular}

Ns: There is no significant difference $(p>0.05)$

$*$ Statistical significant difference $(p<0.05)$

Chi-square test\& Independent samples t-test

Table (2): Percentage distribution of the studied patients regarding to Clinical data $(n=150)$.

\begin{tabular}{|c|c|c|c|c|c|}
\hline \multirow{2}{*}{ Variables } & \multicolumn{2}{|c|}{ Chest ICU $(n=120)$} & \multicolumn{2}{|c|}{ Trauma ICU(n= 30) } & \multirow{2}{*}{ P-value } \\
\hline & No. & $\%$ & No. & $\%$ & \\
\hline \multicolumn{5}{|l|}{ Type respiratory failure } & \multirow{3}{*}{$0.000^{*}$} \\
\hline Type I & 28 & 23.3 & 17 & 56.7 & \\
\hline Type II & 92 & 76.7 & 13 & 43.3 & \\
\hline \multicolumn{5}{|l|}{ Duration of ICU stay: } & \multirow{5}{*}{0.365} \\
\hline$<5$ days & 19 & 15.8 & 3 & 10.0 & \\
\hline $5-<10$ days & 41 & 34.2 & 7 & 23.3 & \\
\hline $10-<15$ days & 32 & 26.7 & 9 & 30.0 & \\
\hline$\geq 15$ days & 28 & 23.3 & 11 & 36.7 & \\
\hline Mean \pm SD & \multicolumn{2}{|c|}{$10.93 \pm 7.59$} & \multicolumn{2}{|c|}{$13.60 \pm 8.54$} & 0.095 \\
\hline \multicolumn{5}{|l|}{ Admission type to ICU } & \multirow{3}{*}{$0.011 *$} \\
\hline First admission & 61 & 50.8 & 23 & 76.7 & \\
\hline Recurrent admission & 59 & 49.2 & 7 & 23.3 & \\
\hline
\end{tabular}

Ns: There is no significant difference $(P>0.05)$

* Statistical significant difference $(p<0.05)$

Chi-square test\& Independent samples t-test 
Table (3): Percentage distribution of the studied patients regarding to past medical and surgical history.

\begin{tabular}{|c|c|c|c|c|c|}
\hline \multirow{2}{*}{ Variables } & \multicolumn{2}{|c|}{ Chest ICU $(n=120)$} & \multicolumn{2}{|c|}{ Trauma ICU $(n=30)$} & \multirow{2}{*}{ P-value } \\
\hline & No. & $\%$ & No. & $\%$ & \\
\hline Hypertension & 59 & 49.2 & 10 & 33.3 & 0.120 \\
\hline DM & 36 & 30.0 & 4 & 13.3 & 0.065 \\
\hline \multicolumn{5}{|l|}{ Previous respiratory disease } & \multirow{3}{*}{$0.000^{*}$} \\
\hline Yes & 116 & 96.7 & 10 & 33.3 & \\
\hline No & 4 & 3.3 & 20 & 66.7 & \\
\hline \multicolumn{6}{|l|}{ Type of respiratory disease } \\
\hline Chronic bronchitis & 40 & 34.5 & 8 & 80.0 & $0.007 *$ \\
\hline COPD & 67 & 57.8 & 2 & 20.0 & $0.042 *$ \\
\hline Pneumonia & 6 & 5.2 & 0 & 0.0 & 1.000 \\
\hline T.B & 3 & 2.6 & 0 & 0.0 & 1.000 \\
\hline Cardiac disease (IHD) & 18 & 15.0 & 0 & 0.0 & $0.024 *$ \\
\hline \multicolumn{5}{|l|}{ Renal disease } & \multirow{3}{*}{0.687} \\
\hline Yes & 9 & 7.5 & 1 & 3.3 & \\
\hline No & 111 & 92.5 & 29 & 96.7 & \\
\hline \multicolumn{6}{|l|}{ Type of renal disease } \\
\hline ARF & 1 & 11.1 & 0 & 0.0 & 1.000 \\
\hline Ectopic kidney & 1 & 11.1 & 0 & 0.0 & 1.000 \\
\hline Renal failure & 4 & 44.4 & 1 & 100.0 & 1.000 \\
\hline Renal impairment & 3 & 33.3 & 0 & 0.0 & 1.000 \\
\hline \multicolumn{5}{|l|}{ Surgical operation } & \multirow{3}{*}{1.000} \\
\hline Yes & 10 & 8.3 & 2 & 6.7 & \\
\hline No & 110 & 91.7 & 28 & 93.3 & \\
\hline \multicolumn{6}{|l|}{ Type of surgical operation } \\
\hline Appendectomy & 2 & 20.0 & 2 & 100.0 & 0.091 \\
\hline Correction of peptic ulcer & 1 & 10.0 & 0 & 0.0 & 1.000 \\
\hline Cut of spinal cord and repair & 1 & 10.0 & 0 & 0.0 & 1.000 \\
\hline Hernia & 1 & 10.0 & 0 & 0.0 & 1.000 \\
\hline Internal fixation & 2 & 20.0 & 0 & 0.0 & 1.000 \\
\hline Nephrectomy & 2 & 20.0 & 0 & 0.0 & 1.000 \\
\hline Thymectomy & 1 & 10.0 & 0 & 0.0 & 1.000 \\
\hline Allergy & 12 & 10.0 & 0 & 0.0 & 0.125 \\
\hline \multicolumn{5}{|l|}{ Smoking status } & \multirow{3}{*}{$0.000 *$} \\
\hline Smoker & 109 & 90.8 & 13 & 43.3 & \\
\hline Non-smoker & 11 & 9.2 & 17 & 56.7 & \\
\hline \multicolumn{5}{|l|}{ Type of smoking } & \multirow{3}{*}{0.215} \\
\hline Current smoker & 69 & 63.3 & 11 & 84.6 & \\
\hline Passive smoker & 40 & 36.7 & 2 & 15.4 & \\
\hline \multicolumn{5}{|l|}{ Smoking index } & \multirow{4}{*}{$0.017^{*}$} \\
\hline Heavy & 48 & 69.6 & 3 & 27.3 & \\
\hline Moderate & 3 & 4.3 & 2 & 18.2 & \\
\hline Mild & 18 & 26.1 & 6 & 54.5 & \\
\hline
\end{tabular}

Ns: There is no significant difference $(p>0.05) *$ Statistical significant difference $(p<0.05) \quad$ Chi-square test \& Independent samples $t$-test 
Table (4): percentage distribution of the studied patients regarding risk factors of acute respiratory failure.

\begin{tabular}{|c|c|c|c|c|c|}
\hline \multirow{2}{*}{ Variables } & \multicolumn{2}{|c|}{ Chest ICU $(n=120)$} & \multicolumn{2}{|c|}{ Trauma ICU $(n=30)$} & \multirow{2}{*}{$\begin{array}{c}\text { P- } \\
\text { value }\end{array}$} \\
\hline & No. & $\%$ & No. & $\%$ & \\
\hline Chronic obstructive pulmonary disease (COPD) & 92 & 76.7 & 7 & 23.3 & $0.000 *$ \\
\hline Asthma & 8 & 6.7 & 3 & 10.0 & 0.460 \\
\hline Cancer of the lung & 4 & 3.3 & 0 & 0.0 & 0.584 \\
\hline Cardiovascular disorders & 25 & 20.8 & 0 & 0.0 & $0.006 *$ \\
\hline Injury of spine, brain or chest & 3 & 2.5 & 15 & 50.0 & $0.000 *$ \\
\hline Family history of respiratory disease & 30 & 25.0 & 3 & 10.0 & 0.076 \\
\hline Smoking & 109 & 90.8 & 13 & 43.3 & $0.000 *$ \\
\hline Inspiratory muscle fatigue & 4 & 3.3 & 0 & 0.0 & 0.584 \\
\hline Allergic disorders & 3 & 2.5 & 1 & 3.3 & 1.000 \\
\hline
\end{tabular}

Ns: There is no significant difference $(p>0.05)$

* Statistical significant difference $(p<0.05)$

Chi-square test \& Independent samples t-test

Table (5): Comparison between both groups in relation to hemodynamic parameters.

\begin{tabular}{|c|c|c|c|c|}
\hline \multirow[t]{2}{*}{ Variable } & \multirow[t]{2}{*}{ hemodynamic parameters } & $\begin{array}{c}\text { Chest ICU } \\
(n=120)\end{array}$ & $\begin{array}{c}\text { Trauma ICU } \\
(n=30)\end{array}$ & \multirow[t]{2}{*}{ P-value } \\
\hline & & Mean \pm SD & Mean \pm SD & \\
\hline \multirow{7}{*}{$1^{\text {st }}$ day } & Heart rate & $93.79 \pm 15.54$ & $105.27 \pm 14.80$ & $0.000^{*}$ \\
\hline & Respiratory rate & $24.54 \pm 3.46$ & $26.83 \pm 3.71$ & $0.003^{*}$ \\
\hline & Temperature & $37.33 \pm 0.29$ & $37.60 \pm 0.38$ & $0.000^{*}$ \\
\hline & Systolic BP & $124.50 \pm 15.17$ & $124.33 \pm 19.42$ & 0.697 \\
\hline & Diastolic BP & $83.25 \pm 58.43$ & $77.33 \pm 12.30$ & 0.613 \\
\hline & MAP & $86.21 \pm 13.90$ & $86.17 \pm 16.28$ & 0.837 \\
\hline & Oxygen saturation & $93.00 \pm 3.78$ & $94.80 \pm 3.24$ & $0.003^{*}$ \\
\hline \multirow{7}{*}{$2^{\text {nd }}$ day } & Heart rate & $97.73 \pm 16.41$ & $107.68 \pm 17.45$ & $0.020^{*}$ \\
\hline & Respiratory rate & $26.18 \pm 3.30$ & $27.55 \pm 5.15$ & 0.098 \\
\hline & Temperature & $37.43 \pm 0.28$ & $37.89 \pm 0.69$ & $0.001 *$ \\
\hline & Systolic BP & $123.79 \pm 13.99$ & $120.00 \pm 13.42$ & 0.439 \\
\hline & Diastolic BP & $77.59 \pm 9.88$ & $76.36 \pm 9.24$ & 0.726 \\
\hline & \begin{tabular}{|l} 
MAP \\
\end{tabular} & $83.71 \pm 12.06$ & $85.00 \pm 11.13$ & 0.777 \\
\hline & \begin{tabular}{|l|} 
Oxygen saturation \\
\end{tabular} & $94.35 \pm 2.13$ & $96.18 \pm 1.71$ & $0.000^{*}$ \\
\hline \multirow{7}{*}{$3^{\text {th }}$ day } & Heart rate & $104.66 \pm 19.53$ & $110.27 \pm 18.70$ & 0.337 \\
\hline & Respiratory rate & $28.97 \pm 3.90$ & $28.27 \pm 2.10$ & 0.608 \\
\hline & Temperature & $37.53 \pm 0.28$ & $37.83 \pm 0.52$ & 0.128 \\
\hline & Systolic BP & $122.74 \pm 12.84$ & $121.20 \pm 16.16$ & 0.615 \\
\hline & Diastolic BP & $77.51 \pm 9.20$ & $77.28 \pm 10.64$ & 1.000 \\
\hline & MAP & $85.69 \pm 12.94$ & $82.73 \pm 11.04$ & 0.380 \\
\hline & Oxygen saturation & $94.07 \pm 2.15$ & $96.00 \pm 1.48$ & $0.011^{*}$ \\
\hline
\end{tabular}

MAP: mean arterial pressure

Chi-square test 
Table (6): Comparison between both groups in relation to arterial blood gases\&oxygen saturation.

\begin{tabular}{|c|c|c|c|c|}
\hline & \multirow{2}{*}{ arterial blood gases } & Chest ICU $(n=120)$ & Trauma ICU $(n=30)$ & \multirow{2}{*}{ P-value } \\
\hline & & Mean \pm SD & Mean \pm SD & \\
\hline \multirow{7}{*}{$1^{\text {st }}$ day } & PH & $7.33 \pm 0.11$ & $7.38 \pm 0.12$ & 0.067 \\
\hline & $\mathrm{PaCo}_{2}$ & $63.31 \pm 20.80$ & $46.97 \pm 22.94$ & $0.000 *$ \\
\hline & $\mathrm{HCO}_{3}$ & $32.42 \pm 8.77$ & $28.76 \pm 9.60$ & $0.041 *$ \\
\hline & \begin{tabular}{|l|}
$\mathrm{SaO}_{2}$ \\
\end{tabular} & $90.18 \pm 7.38$ & $93.80 \pm 4.72$ & $0.003^{*}$ \\
\hline & $\mathbf{B E}$ & $-9.00 \pm 0.00$ & $-4.68 \pm 4.47$ & $0.043 *$ \\
\hline & $\mathrm{PaO}_{2}$ & $65.98 \pm 22.07$ & $75.80 \pm 24.42$ & $0.029 *$ \\
\hline & $\mathrm{O}_{2}$ saturation & $90.33 \pm 7.26$ & $93.80 \pm 4.72$ & $0.004 *$ \\
\hline \multirow{7}{*}{$2^{\text {nd }}$ day } & PH & $7.42 \pm 0.07$ & $7.46 \pm 0.05$ & $0.008 *$ \\
\hline & \begin{tabular}{|l|}
$\mathrm{PaCo}_{2}$ \\
\end{tabular} & $56.44 \pm 13.34$ & $44.45 \pm 12.94$ & $0.000 *$ \\
\hline & $\mathrm{HCO}_{3}$ & $36.52 \pm 8.85$ & $30.45 \pm 7.16$ & $0.004 *$ \\
\hline & $\mathrm{SaO}_{2}$ & $94.26 \pm 2.22$ & $96.50 \pm 1.65$ & $0.000 *$ \\
\hline & $\mathbf{B E}$ & $-5.50 \pm 3.54$ & $-0.20 \pm 4.85$ & 0.399 \\
\hline & $\mathrm{PaO}_{2}$ & $70.09 \pm 10.48$ & $86.41 \pm 23.16$ & $0.004 *$ \\
\hline & $\mathrm{O}_{2}$ saturation & $94.27 \pm 2.22$ & $96.55 \pm 1.63$ & $0.000 *$ \\
\hline \multirow{7}{*}{$3^{\text {th }}$ day } & $\mathbf{P H}$ & $7.44 \pm 0.05$ & $7.45 \pm 0.05$ & 0.501 \\
\hline & $\mathrm{PaCo}_{2}$ & $59.96 \pm 13.78$ & $43.00 \pm 10.30$ & $0.001 *$ \\
\hline & $\mathrm{HCO}_{3}$ & $39.09 \pm 8.73$ & $31.05 \pm 6.41$ & $0.009 *$ \\
\hline & $\mathrm{SaO}_{2}$ & $93.68 \pm 2.92$ & $96.27 \pm 1.49$ & $0.004 *$ \\
\hline & \begin{tabular}{|l|}
$\mathbf{B E}$ \\
\end{tabular} & $-1.57 \pm 0.14$ & $-1.65 \pm 0.13$ & 0.432 \\
\hline & \begin{tabular}{|l|}
$\mathbf{P a O 2}$ \\
\end{tabular} & $71.46 \pm 7.47$ & $95.00 \pm 14.50$ & $0.000 *$ \\
\hline & $\mathbf{O}_{2}$ saturation & $93.68 \pm 2.74$ & $96.27 \pm 1.49$ & $0.003 *$ \\
\hline
\end{tabular}

Ns: There is no significant difference $(p>0.05) *$ Statistical significant difference $(p<0.05)$

ABG: arterial blood gas

$\mathrm{PaCO}_{2}$ : partial pressure of carbon dioxide

$\mathrm{PaO}_{2}$ : partial pressure of oxygen $\quad \mathrm{Hco}_{3}$ : bicarbonate $\mathrm{BE}$ : base deficit $\quad \mathrm{SaO}_{2}$ : oxygen saturation

Chi-square test

Table (7): Comparison between both groups in relation to mechanical ventilator parameters.

\begin{tabular}{|c|c|c|c|c|}
\hline & \multirow{2}{*}{ ventilatory parameters } & Chest ICU $(n=120)$ & Trauma ICU $(n=30)$ & \multirow{2}{*}{ P-value } \\
\hline & & Mean \pm SD & Mean \pm SD & \\
\hline \multirow{5}{*}{$\mathbf{1}^{\text {st }}$ day } & Respiratory rate & $12.01 \pm 2.38$ & $14.00 \pm 2.99$ & $0.000 *$ \\
\hline & Pressure support & $14.90 \pm 1.33$ & $16.50 \pm 2.73$ & $0.000 *$ \\
\hline & Fio2 & $45.86 \pm 12.17$ & $46.17 \pm 9.07$ & 0.403 \\
\hline & PEEP & $5.28 \pm 1.05$ & $6.67 \pm 2.14$ & $0.000 *$ \\
\hline & Tidal volume (VT) & $500.71 \pm 38.32$ & $500.00 \pm 43.30$ & 0.850 \\
\hline \multirow{5}{*}{$2^{\text {nd }}$ day } & Respiratory rate & $11.83 \pm 1.69$ & $15.35 \pm 2.69$ & $0.000 *$ \\
\hline & \begin{tabular}{|l|} 
Pressure support \\
\end{tabular} & $15.17 \pm 1.85$ & $17.54 \pm 2.50$ & $0.001^{*}$ \\
\hline & Fio2 & $41.20 \pm 7.81$ & $48.42 \pm 9.58$ & $0.001 *$ \\
\hline & PEEP & $5.63 \pm 1.18$ & $6.53 \pm 2.22$ & 0.052 \\
\hline & \begin{tabular}{|l} 
Tidal volume (VT) \\
\end{tabular} & $502.67 \pm 32.26$ & $495.29 \pm 88.33$ & 0.955 \\
\hline \multirow{5}{*}{$3^{\text {th }}$ day } & Respiratory rate & $11.35 \pm 1.37$ & $16.36 \pm 2.80$ & $0.000 *$ \\
\hline & Pressure support & $14.71 \pm 2.41$ & $16.14 \pm 2.04$ & 0.125 \\
\hline & Fio2 & $42.85 \pm 10.61$ & $51.82 \pm 14.88$ & 0.067 \\
\hline & PEEP & $6.10 \pm 1.41$ & $5.91 \pm 1.22$ & 0.860 \\
\hline & Tidal volume (VT) & $511.82 \pm 25.23$ & $500.91 \pm 49.08$ & 0.516 \\
\hline
\end{tabular}

MV: mechanical ventilation

PEEP: Positive end expiratory pressure

PS: Pressure support RR: Respiratory rate Vt: Tidal volume

Ns: There is no significant difference $(p>0.05)$

* Statistical significant difference $(p<0.05)$

Chi-square test

Vol , (7) No , (17) June, 2019 
Table (1): Represents percentage distribution of studied patients regarding patient's characteristics. It was noticed that $56.7 \%$ of patients in trauma ICU aged $<40$ years versus $37.5 \%$ in chest ICU were aged 40 - <50 years, with statistical significant differences between both groups $(\mathrm{p}=0.003 *)$. The mean age of patients in chest ICU groups were $51.16 \pm 9.17$ years versus $42.87 \pm 11.74$ years in the trauma ICU groups with statistical significant differences respectively. Regarding to sex, It was found that $70 \%$ in the trauma ICU groups were male versus $60.8 \%$ in the chest ICU groups. Regarding to occupation, it was founds that $35.8 \%$ in the chest ICU groups were house wife versus $33.3 \%$ of the trauma ICU groups were Unemployed, with statistical significant differences between both groups ( $\left.p=0.000^{*}\right)$. Regarding to Marital status it was noticed that $97.5 \%$ in chest ICU groups were married versus $93.3 \%$ in the trauma ICU groups. With no statistical significant differences between both groups.

Table (2): Shows percentage distribution of studied patients regarding to clinical data it was found that $76.7 \%$ in the chest ICU groups were Type II versus $56.7 \%$ of the trauma ICU groups were Type I, with statistical significant differences between both groups $\left(\mathrm{p}=0.000^{*}\right)$. Regarding to duration in ICU, it was found that the mean duration of patients in chest ICU groups were $(10.93 \pm 7.59)$ versus $(13.60 \pm 8.54)$ in the trauma ICU groups with no statistical significant differences. Regarding to type of admission to ICU, it was founds that $76.7 \%$ of the trauma ICU groups were first admission versus $50.8 \%$ in the chest ICU groups, with statistical significant differences between both groups $(\mathrm{p}=0.011 *)$.

Table (3): Showed percentage distribution of studied patients regarding to past medical and surgical history, founded that; less than half of both groups had a history of hypertension and diabetes mellitus, it was found that nearly all of patients in the chest ICU groups suffered from respiratory disease versus one third in the trauma ICU groups had a history of respiratory disease, with statistical significant differences between both groups $(\mathrm{p}=0.000 *)$. Regarding to smoking status it was found that $90.8 \%$ in the chest ICU groups were smoker versus $56.7 \%$ of the trauma ICU groups were non-smoker, it was noticed high percentage of them $84.6 \%$ were current smokers, Also, it was found that $69.6 \%$ of current smoker were heavy smokers there were statistical significant differences between both groups regarding respiratory disease, cardiac disease and smoking $(\mathrm{p}=$ $0.000 *, 0.024 *)$.

Table (4): Showed percentage distribution of studied patients regarding to risk factors it was noticed that $90.8 \%$ in the chest ICU groups were smoking and $76.7 \%$ were suffer from COPD but Injury of spine, brain or chest and smoking were the majority risk factors in the trauma ICU groups (50\% and 43,3\% respectively). With statistical significant differences between both groups $\left(\mathrm{p}=0.000^{*}\right)$. There were statistical significant differences between chest ICU groups and trauma ICU groups related to COPD, Injury of spine, brain or chest, smoking and cardiovascular disorders $\mathrm{p}$ = $(0.000 * \& 0.006 *)$ respectively.

Table (5): Shows vital signs and hemodynamic parameters of chest ICU groups and trauma ICU groups. At first day, it was noticed statistical significant difference between chest ICU groups and trauma ICU groups related to heart rate, respiratory rate, temperature and oxygen saturation $\left(\mathrm{p}=0.000^{*}\right.$, $0.003 *)$ respectively. At second day, it was noticed statistical significant difference between chest ICU groups and trauma ICU patients related to heart rate, temperature and oxygen saturation (p $\left.=0.020^{*}, 0.001^{*}, 0.000^{*}\right)$. At third day, it was noticed statistical significant difference between chest ICU groups\& trauma ICU patients related to oxygen saturation only $\left(\mathrm{p}=0.011^{*}\right)$.

Table (6): The table enumerate that arterial blood gas parameter of chest ICU groups and trauma ICU groups. At first day, it was noticed statistical significant difference between chest ICU groups and trauma ICU groups related to $\mathrm{PaCo}_{2}, \mathrm{HCO}_{3}, \mathrm{SaO}_{2}$, $\mathrm{BE}, \mathrm{PaO}_{2}$ and $\mathrm{O}_{2}$ saturation $(\mathrm{p}=<0.05)$. At second day, it was noticed that there was statistical significant difference between chest ICU groups\& trauma ICU patients related to $\mathrm{PH}, \mathrm{PaCo}_{2}, \mathrm{HCO}_{3}$, $\mathrm{SaO}_{2}, \mathrm{PaO}_{2}$ and $\mathrm{O}_{2}$ saturation $(\mathrm{p}=<0.05)$. At third day, it was noticed that there was statistical significant difference between chest ICU groups\& trauma ICU patients related to $\mathrm{PaCo}_{2}, \mathrm{HCO}_{3}, \mathrm{SaO}_{2}$, $\mathrm{PaO}_{2}$ and $\mathrm{O}_{2}$ saturation $(\mathrm{p}=<0.05)$.

Table (7): Represents comparison between the chest ICU groups and trauma ICU groups as regard to mechanical ventilator parameters. At first day, it was noticed statistical significant difference between chest ICU groups and trauma ICU groups related to RR, PS and PEEP $(p=0.000 *)$ respectively, At second day, it was noticed statistical significant difference between chest ICU groups \& trauma ICU patients related to RR, PS and $\mathrm{Fio}_{2}\left(\mathrm{p}=0.000^{*}\right.$, $0.001 *)$ respectively. At third day, it was noticed that there was statistical significant difference between chest ICU groups \& trauma ICU patients related to $\operatorname{RR}\left(\mathrm{p}=0.000^{*}\right)$. But here were no statistical difference between both groups regarding PEEP and VT in fist and third day.

\section{Discussion}

The presence of ARF unembellished enough on necessitate mechanical ventilator sustenance is seen 
by some as critical to the development of intensive care medicine as a specialty in its own right. Acute respiratory failure (ARF) in critically ill patients is still accompanying with mortality rates of between $40 \%$ and $65 \%$.Most of the available literature has attentive on the severest form of ARF, namely, ARDS, and scarce studies have weighed either the incidence of mortality rate of ARF in general between critically ill patients (Culnan et al., 2018). Therefore, the present study aimed to identify prevalence and risk factors of Acute respiratory failure among critically ill patients.

This discussion will cover the main result findings as follow:

\section{Patient's characteristics of studied group}

Based on the results of the present study; more than half of study patients in trauma ICU aged less than fourteen years versus from less than half were from fourteen to less than fifteen years old in chest ICU, The mean age of patients in chest ICU groups were $51.16 \pm 9.17$ years versus $42.87 \pm 11.74$ years in the trauma ICU groups with statistical significant differences respectively with statistical significant differences $\left(\mathrm{p}=0.003^{*}\right)$. This not agreed with Cameron et al., (2016) who found the age of ICU patients were more than 50 years old. Also this not agreed with Barry \& Thompson, (2018) found that patients mean age was 67.5 years old. Prior research has shown that severity of illness is more important than age on patients' prognosis, and aggressive treatment strategies are not less cost-effective when provided to older patients.

Phua et al., (2009) concluded that the only factors associated with mortality changes were patient age. Although age is a consistent risk factor for the incidence of ARF.

Most of patients in the both chest ICU groups and trauma ICU groups were male. This compatable with Flaatten et al., (2017) who found no significant difference between both sex which compatable with the result of the current study. In contrast GarnachoMontero et al., (2018) found that male: female ratio in admitted ICU patients suffering from ARF was 1:2 In my opinion the most of patient was male related to smoking and work environment.

Regarding to occupation, it was founds that the large number of studied sample in the chest ICU groups were house wife versus more than one third of the trauma ICU groups were not work, with statistical significant differences between both groups $\left(\mathrm{p}=0.000^{*}\right)$. It was noticed that majority in both groups were married, with no statistical significant differences between both groups. This compatable with (Ely, 2017).

\section{Regarding Clinical data of patients:}

The result of the present study showed that; majority of studied patients in the chest ICU groups were Type II ARF versus more than half of the trauma ICU groups were type I ARF, This match to the result of the study done by Garrouste- Orgeas et al., (2018) who mentioned that Hypercapnic respiratory failure (type 2) in which paco $_{2}>50 \mathrm{mmHg}$, is result of scant alveolar ventilation subordinate to decreased ventilator drive, respiratory muscle fatigue or failure and increased work of breathing.

Regarding to duration in ICU, the findings revealed that the mean duration of patients in chest ICU groups were from 5 to 10 days.This was not in the same line with Burgos et al., (2011), but in opposite founding Weinberger et al., (2010) revealed that; the rang of patient stay in ICU ranged from 9 to 55 days.

Regarding to type of admission to ICU, it was founds that majority of the trauma ICU groups were first admission but only around half of the patients in the chest ICU groups, This in the same line with Sikter, (2016) found that, major diagnoses at admission were metabolic problems, refeeding survey and voluntary drug intoxication and infection. Where $60 \%$ of the readmitted in the ICU for the same reason.

The present study demonstrated regarding past medical and surgical history, It founded that; The less than half of both groups had a history of hypertension and diabetes mellitus, it was found that nearly all of patients in the chest ICU groups suffered from respiratory disease versus one third in the trauma ICU groups had a history of respiratory disease, This in compatible with (Ramesh et al., (2018).

This in the same line with, Sarkar et al., (2017) indicated that the risk factors for acute respiratory failure were highly with COPD, Injury of spine, brain or chest, smoking and cardiovascular disorders.

Regarding to smoking status it was found that most of studied patients admitted in the chest ICU groups were smoker versus near half in the trauma ICU groups, it was noticed high percentage of them were current smokers, Also, it was found that two third of current smoker were heavy smokers there were statistical significant differences between both groups regarding respiratory disease, cardiac disease and smoking .

It is perhaps not surprising that smoking is linked to ARF through an increased incidence of pneumonia; however, this remembrance persists even in patients who are at risk from non-pulmonary sources and when smoking history is gained biochemically (Calfee et al., 2015)

Risk factors of acute respiratory failure The finding of the present study showed that the majority risk factors among studied sample in the chest ICU groups were smoking and COPD versus 
majority in the trauma ICU groups was Injury of spine, brain or chest, with statistical significant differences between both groups $(\mathrm{p}=0.000 *)$. There were statistical significant differences between chest ICU groups and trauma ICU groups related to COPD, Injury of spine, brain or chest, smoking and cardiovascular disorders $\mathrm{p}=(0.000 * \& 0.006 *)$ respectively. Indicating that the risk factors for acute respiratory failure were highly with COPD, Injury of spine, brain or chest, smoking and cardiovascular disorders. This finding not matched with (Standiford \& Ward (2016) who founded that common risk factors for ARF include sepsis, aspiration of gastric contents, shock, and infection. Where De Prost et al., (2017) founded that COPD is the main risk factor of ARF

Regarding vital signs \& hemodynamic parameters of chest ICU groups and trauma ICU groups.

At first day, it was noticed statistical significant difference between chest ICU groups and trauma ICU groups related to heart rate, respiratory rate, temperature and oxygen saturation ( $\mathrm{p}=0.000^{*}$, $\left.0.003^{*}\right)$ respectively. At first day, it was noticed statistical significant difference between chest ICU groups \& trauma ICU patients related to heart rate, temperature and oxygen saturation ( $\mathrm{p}$ $=0.020 *, 0.001 *, 0.000 *)$. At third day, it was noticed statistical significant difference between chest ICU groups \& trauma ICU patients related to oxygen saturation only $(\mathrm{p}=0.011 *)$.

The finding of the current study was not matched with Mowery, (2017) who found that; major criteria included respiratory arrest, respiratory pause with loss of consciousness, severe hemodynamic instability (heart rate $<50$ beats/min with loss of alertness, and/or systolic blood pressure $<70 \mathrm{~mm}$ $\mathrm{Hg}$ ). Minor criteria were a RR 35 breaths/min and above the value on admission, PaO 2 : F IO 2 150, an increase in $\mathrm{Pa} \mathrm{CO} 2.20 \%$ from prior arterial blood gas measurement, and variation in mental station attributable to respiratory damage.

Regarding arterial blood gas parameter of chest ICU groups \& trauma ICU groups.

At first day, it was noticed statistical significant difference between chest ICU groups and trauma ICU groups related to $\mathrm{PaCo}_{2}, \mathrm{HCO}_{3}, \mathrm{SaO}_{2}, \mathrm{BE}, \mathrm{PaO}_{2}$ and $\mathrm{O}_{2}$ saturation ( $\mathrm{p}=<0.05)$. At second day, it was noticed statistical significant difference between chest ICU groups \& trauma ICU patients related to $\mathrm{PH}, \mathrm{PaCo}_{2}$, , $\mathrm{HCO}_{3}, \mathrm{SaO}_{2}, \mathrm{PaO}_{2}$ and $\mathrm{O}_{2}$ saturation $(\mathrm{p}=<0.05)$. At third day, it was noticed statistical significant difference between chest ICU groups \& trauma ICU patients related to $\mathrm{PaCO}_{2}, \mathrm{HCO}_{3}, \mathrm{SaO}_{2} \quad \mathrm{PaO}_{2}$ and $\mathrm{O}_{2}$ saturation $(\mathrm{p}=<0.05)$.

The study by Funk et al., (2013) mentioned that the patients suffer from ARF had hypercapnia episode similar efficiency as for subjects with COPD. In addition, Lun et al., (2016) founded in their study that; A proportion of patients with more severe disease will have a respiratory acidosis $(\mathrm{pH}<7.35$ and $\mathrm{PaCO} 2>6 \mathrm{k} \mathrm{Pa}$ ) as a result of acute on chronic respiratory failure. Acidosis is associated with augmented mortality also a developed need for intubation

Rochwerg et al., (2017) recommended that all patients with COPD with a respiratory acidosis $(\mathrm{pH}$ $<7.35$ ) should receive NIV, and the British Thoracic Society published guidelines which recommended that NIV should be given to those with a $\mathrm{pH}$ of $<7.25$ and should be painstaking for those with a $\mathrm{pH}$ between 7.25 and 7.35.

In addition Butler et al., (2016) founded that $(\mathrm{PaO} 2)$ in the $47 \%$ of patients who were hypercapnia, with a $\mathrm{PaO} 2$ of $>10 \mathrm{kPa}$ being associated with acidosis in most hypercapnia patients. $80 \%$ persisted acidotic after initial treatment.

In patients with acute $\mathrm{ARF}$ the $\mathrm{PaO} 2$ should be maintained at $7.3-10 \mathrm{kPa}(\mathrm{SaO} 285-92 \%)$ to avoid the dangers of hypoxia and acidosis. If all COPD patients with a respiratory acidosis $(\mathrm{pH}<7.35)$ after primary treatment. However, to affect current practice it will also be essential to provide a lower limit of tolerable $\mathrm{PaO} 2$. There is some suggestion from the data that acidosis is minimized in the $\mathrm{PaO} 2$ range $7.3-10 \mathrm{kPa}$. A lower limit of $\mathrm{PaO} 2$ of $7.3 \mathrm{kPa}$ also provides consistency with the current recommendations for long term oxygen therapy. This range of $\mathrm{PaO} 2(7.3-10 \mathrm{kPa})$ equates to an $\mathrm{SaO} 2$ of 85-92\%. (Tomasic et al., 2018).

Targeting oxygen supplementation on $\mathrm{SaO} 2$ or $\mathrm{PaO} 2$ rather than by $\mathrm{FiO} 2$ may reduce the incidence of oxygen induced acidosis, but this necessitates confirmation in future studies. (Monsieurs et al., 2015).

In the comparison between the chest ICU groups and trauma ICU groups as regard to mechanical ventilator parameters

At first day, it was noticed statistical significant difference between chest ICU groups and trauma ICU groups related to RR, PS and $\operatorname{PEEP}\left(\mathrm{p}=0.000^{*}\right)$ respectively, At second day, it was noticed statistical significant difference between chest ICU groups \& trauma ICU patients related to RR, PS and Fio2 (p $\left.=0.000^{*}, 0.001 *\right)$ respectively. At third day, it was noticed that there was statistical significant difference between chest ICU groups \& trauma ICU patients related to $\mathrm{RR}\left(\mathrm{p}=0.000^{*}\right)$.

Pisani et al., (2016) discussed the best evidencedbased conventional protective targets (i.e. tidal volume $<6 \mathrm{~mL} \cdot \mathrm{kg}-1$ predicted body weight, pressure plateau $<30 \mathrm{cmH} 2 \mathrm{O}$, driving pressure $<15 \mathrm{cmH} 2 \mathrm{O}$ ) and alveolar recruitment options (e.g. higher positive 
end-expiratory pressure (PEEP) levels) in ARDS patients. However, the auspicious influence of this protective strategy on clinical outcomes is not acceptable in all clinical and physiological ARDS scenarios. Accordingly, the authors stress the concept that some ventilator strategies are likely to work in some subsets of patients while they could be unproductive or even detrimental in others. Less than two-thirds of patients with ARDS received protective lung ventilation; plateau pressure was measured in only $40.1 \%$ of the cases whereas $<20 \%$ of patients customary PEEP levels $>12 \mathrm{cmH} 2 \mathrm{O}$

Similarly, On the contrary, the Gajic study found higher airway pressures (peak inspiratory, plateau, and driving pressures), lower PEEP, and higher respiratory rate accompanying with higher hospital mortality, along with older age and ARF.( Gajic et al., 2011)

Carteaux et al., (2016) revealed the factors related hyperventilatory pattern in severely hypoxemic patients as was suggested by the correlation found between tidal volume values $>9.5 \mathrm{~mL} \cdot \mathrm{kg}-1$ predicted body weight and mortality rate in noninvasively ventilated ARF.

Narendra et al., (2017) founded that; less than twothirds of patients with ARF received protective lung ventilation; plateau pressure was measured in solitary $40.1 \%$ of the cases whereas $<20 \%$ of patients received PEEP levels $>12 \mathrm{cmH} 2 \mathrm{O}$.

\section{Conclusion}

The present study conducted that, incidence of acute respiratory failure among critically ill Patients at Assiut University Hospital over six months There was statistical significant differences between chest ICU groups and trauma ICU groups related to COPD, Injury of spine, brain or chest, smoking and cardiovascular disorders were the major risk factors for ARF $\mathrm{p}=\left(0.000^{*} \& 0.006^{*}\right)$ respectively. There was a high statistical significant difference between both groups (chest ICU groups \& trauma ICU groups) regarding their age and Occupation, There was a high statistical significant difference between both groups (chest ICU groups \& trauma ICU groups) regarding patient's outcome.

\section{Recommendations}

The study recommended that

1. Screening of those living in rural area for ARF.

2. Application of life style modification for modifying risk factors leading to ARF.

3. Nures must receive adequate knowledge on signs and symptoms of acute respiratory failure for early detection and management.

\section{Reference}

1. Burgos J., Lujan M, Falco' V., (2011): The spectrum of pneumococcal empyema in adults in the early 21 st century. Clin Infect Dis; 53: 254-261.

2. Butler, A., Smith, L., \& Mackay, A., (2016): Acute exacerbations of COPD. Pulmonary Emergencies (ERS Monograph). Sheffield, European Respiratory Society, 48-65.

3. Calfee C., Matthay M., Kangelaris K., Siew E., Janz D., Bernard G., May A., Jacob P., Havel C., Benowitz N., (2015): Cigarette smoke exposure and the acute respiratory distress syndrome. Crit Care Med 2015;43:1790-1797.

4. Carteaux G., Millán- Guilarte T., \& De Prost N., (2016): Failure of noninvasive ventilation for de novo acute hypoxemic respiratory failure. Crit Care Med, 44: 282-290.

5. Chiumello D., Brochard L., Marini J., (2017): Respiratory support in patients with acute respiratory distress syndrome: an expert opinion. Critical Care; P.P.21: 240

6. Craig D., Stephen B., Mark E., Daniel K., Colin G., Alastair G., (2016): BTS/ICS guideline for the ventilatory management of acute hypercapnic respiratory failure in adults FREE, BMJ Open Respiratory Research journal, 71(2).

7. Culnan, D., Sherman, W., Chung, K., \& Wolf, S., (2018): Critical Care in the Severely Burned: Organ Support and Management of Complications. In Total Burn Care (pp. 328354). Elsevier.

8. Fernández-Barat, L., Ferrer, M., De Rosa, F., Gabarrús, A., Esperatti, M., Terraneo, S., \& Torres, A., (2017): Intensive care unit-acquired pneumonia due to Pseudomonas aeruginosa with and without multidrug resistance. Journal of Infection, 74(2), 142-152.

9. Flaatten, H., De Lange, D., Artigas, A., Bin, D., Moreno, R., Christensen, S., \& Soares, M., (2017): The status of intensive care medicine research and a future agenda for very old patients in the ICU. Intensive care medicine, 43(9), 1319-1328

10. Funk G., Bauer P., Burghuber O., Fazekas A., Hartl S., Hochrieser H., (2013): Prevalence and prognosis of COPD in critically ill patients between 1998 and 2008. Eur Respir J;41(4):792-799.

11. Gajic O., Dabbagh O., Park P., Adesanya A., Chang S., Hou P., Anderson H., Hoth J., Mikkelsen M., Gentile N., (2011): U.S. Critical Illness and Injury Trials Group: Lung Injury Prevention Study Investigators (USCIITG- 
LIPS). Early identification ofpatients at risk of acute lung injury: evaluation of lung injury prediction score in a multicenter cohort study. Am J Respir Crit Care Med;183: 462-470.

12. Garnacho-Montero, J., León-Moya, C., Gutiérrez-Pizarraya, A., ArenzanaSeisdedos, A., Vidaur, L., Guerrero, J., (2018): on Behalf GETGAG Study Group. Clinical characteristics, evolution, and treatment-related risk factors for mortality among immunosuppressed patients with influenza A (H1N1) virus admitted to the intensive care unit. Journal of critical care, 48, 172-177.

13. Garrouste-Orgeas, M., Azoulay, E., Ruckly, S., Schwebel, C., de Montmollin, E., Bedos, J., \& Dumenil, A., (2018): Diabetes was the only comorbid condition associated with mortality of invasive pneumococcal infection in ICU patients: a multicenter observational study from the Outcomerea research group. Infection, 46(5), 669-677.

14. Leclair, T., \& Allen, G., (2018): Acute respiratory failure/acute respiratory distress syndrome. Critical Care Secrets, 188.

15. Lun, C., Tsui, M., Cheng, S., Chan, V., Leung, W., Cheung, A., \& Chu, C., (2016): Differences in baseline factors and survival between normocapnia, compensated respiratory acidosis and decompensated respiratory acidosis in COPD exacerbation: A pilot study. Respirology, 21(1), 128-136.

16. Malay S., Niranjan N., Banyal P., (2017): Mechanisms of hypoxemia, Lung India | Published by Wolters Kluwer, 34(1):47-60.

17. Manzano F., Yuste E., Colmenero M., Aranda A., Garc' 1a-Horcajadas A., Rivera R., Fern'andez- Mond'ejar E., (2005): Granada Respiratory Failure Study Group. Incidence of acute respiratory distress syndrome and its relation to age. J Crit Care;20:274-280.

18. Mc Donell, W., \& Kerr, C., (2015): Physiology, Pathophysiology, and Anesthetic Management of Patients with Respiratory. Veterinary anesthesia and analgesia: the fifth edition of Lumb and Jones,P.P. 513.

19. Michelle F., (2014): Caring for patients in respiratory failure, american nurse today, 9(11).

20. Mowery, N., (2017): Ventilator strategies for chronic obstructive pulmonary disease and acute respiratory distress syndrome. Surgical Clinics, 97(6), 1381-1397

21. Narendra D., Hess D., Sessler C., (2017): Update in management of severe hypoxemic respiratory failure. Chest; 152: 867-879
22. patricia G., \& Dorrie K., (2018): critical care nursing: A Holistic Approach, $11^{\text {th }}$ ed, Wolters Kluwer Health | Lippincott Williams\& Wilkins.

23. Pfister, G., Burkes, R., Guinn, B., Steele, J., Kelley, R., Wiemken, T., \& Cavallazzi, R., (2016): Opioid overdose leading to intensive care unit admission: epidemiology and outcomes. Journal of critical care, 35, 29-32.

24. Phuong V., \& Virginia S., (2014): Respiratory Failure, journal of American Academy of Pediatrics, 35(11).

25. Pisani L., Corcione N., Nava S., (2016): Management of Acute Hypercapnic Respiratory Failure. Curr Opin Crit Care 2016; 22: 45-52

26. Pradeep K., Ghansham B., Sadik M., Priyanka S., \& Pooja B., (2016): Acute respiratory failure and mechanical ventilation in pregnant patient: A narrative review of literature, Journal of Anaesthesiology Clinical Pharmacology, 32(4): 431-439.

27. Rahul M., Shobitha R., Bharti C., Ramkumar V., Hameed A., \& Umesh G., (2017): Epidemiological profile of acute respiratory distress syndrome patients: A tertiary care experience, lung India, Indian Chest Society,34(1):471-484.

28. Ramesh, H., Krishnan, J., \& Manikath, N., (2018): keywords sepsis, acute kidney injury, akin criteria, hypertension, diabetes mellitus. acute kidney injury in sepsis patientsan observational study, p.97: 99.

29. Rochwerg, B., Brochard, L., Elliott, M., Hess, D., Hill, N., Nava, S., \& Ferrer, M., (2017): Official ERS/ATS clinical practice guidelines: noninvasive ventilation for acute respiratory failure. European Respiratory Journal, 50(2), 1602426

30. Rogerson, D., Williams, J., Yates, S., \& Rogers, E., (2017): Epidemiology, practice of ventilation and outcome for patients at increased risk of postoperative pulmonary complications. European Journal of Anaesthesiology, 34(8), 492-507

31. Sarkar, M., Niranjan, N., \& Banyal, P., (2017): Mechanisms of hypoxemia. Lung India: Official Organ of Indian Chest Society, 34(1), 47.

32. Standiford, T., \& Ward, P., (2016): Therapeutic targeting of acute lung injury and acute respiratory distress syndrome. Translational Research, 167(1), 183191.

33. Sikter, A., (2016): Modeling of the Cytoplasm. English version) Card. Hung. Suppl. B, 37, 3-47. 
34. Tomasic, I., Tomasic, N., Trobec, R., Krpan, M., \& Kelava, T., (2018): Continuous remote monitoring of COPD patients-justification and explanation of the requirements and a survey of the available technologies. Medical \& biological engineering \& computing, 1-23

35. Weinberger D., Harboe Z., Sanders E., (2010): Association of serotype with risk of death due to pneumococcal pneumonia: a metaanalysis. Clin Infect Dis; 51: 692-699. 